\title{
Strange Hadron Production from STAR Fixed-Target Program
}

\section{Muhammad Usman Ashraf*广 (for the STAR Collaboration)}

Department of Engineering Physics, Tsinghua University, Beijing 100084, China

E-mail: musman_mughal10@yahoo.com

\begin{abstract}
We report the transverse mass spectra and yield $(d N / d y)$ for strange hadrons $\left(\mathrm{K}_{\mathrm{S}}^{0}\right.$ and $\left.\Lambda\right)$ in rapidity interval of $0.25<|y|<1.75$ from the first dedicated run of the STAR Fixed-Target program in 2015 with $\mathrm{Au}+\mathrm{Au}$ collisions at $\sqrt{\mathrm{s}_{\mathrm{NN}}}=4.5 \mathrm{GeV}$ and $\mathrm{Al}+\mathrm{Au}$ at $\sqrt{\mathrm{s}_{\mathrm{NN}}}=4.9 \mathrm{GeV}$. These results are compared with the previous published results from different AGS experiments. The reasonable agreements demonstrate that STAR has good event reconstruction and particle identification capabilities in fixed-target configuration. The physics implications for collision dynamics of these results and the future STAR Fixed-Target program are discussed.
\end{abstract}

Critical Point and Onset of Deconfinement - CPOD2017

7-11 August, 2017

The Wang Center, Stony Brook University, Stony Brook, NY

\footnotetext{
* Speaker.

${ }^{\dagger}$ A footnote may follow.
} 


\section{Introduction}

Ultra-relativistic heavy-ion collisions are the most promising tool for the creation of a new state of matter where partonic interactions dominate, referred as the Quark-Gluon Plasma (QGP). According to Lattice QCD calculations [1], the critical conditions for the QGP phase transition are reached at a critical temperature $T_{c} \sim 150 \mathrm{MeV}$. STAR has observed a QGP state in $\mathrm{Au}+\mathrm{Au}$ collisions at the top RHIC energy of $\sqrt{\mathrm{s}_{\mathrm{NN}}}=200 \mathrm{GeV}$ [2]. The RHIC Beam Energy Scan (BES) was proposed to search for the QCD critical point and study the nature of the phase transition between partonic and hadronic matter [3]. Figure 1 shows a wide range of temperatures, $T$, and baryon chemical potentials, $\mu_{B}$, in the QCD phase diagram can be accessed by systematically studying the $\mathrm{Au}+\mathrm{Au}$ collisions from $\sqrt{\mathrm{s}_{\mathrm{NN}}}=200 \mathrm{GeV}$ down to $7.7 \mathrm{GeV}$. The data from the NA49 experiment at CERN suggest that the onset of deconfinement may occur at $\sqrt{\mathrm{s}_{\mathrm{NN}}} \approx 7 \mathrm{GeV}$, which is the low end of BES range [4].

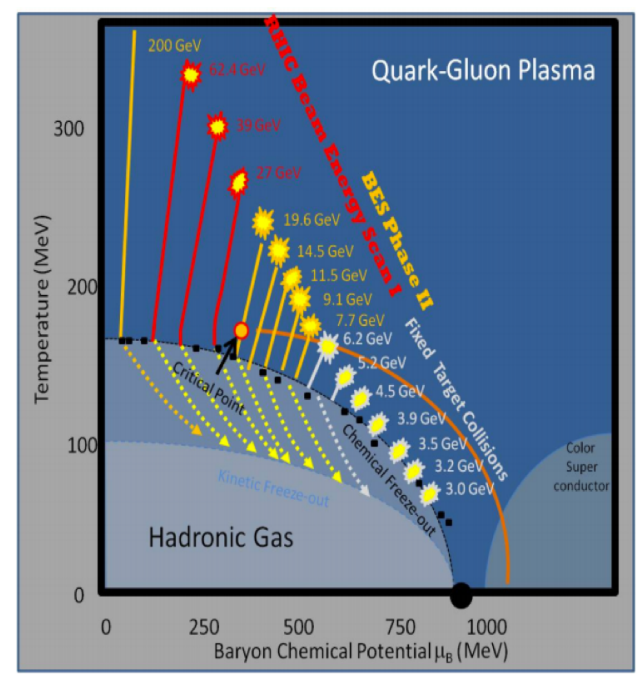

Figure 1: QCD phase diagram as a function of temperatures, $T$, and baryon chemical potentials, $\mu_{B}$, with indication of the BES and Fixed-Target (FXT) program ranges.

Strange particle production provides one of the most powerful tools to study the dynamics of the reaction. Particularly, an enhancement in strange particle production in $\mathrm{A}+\mathrm{A}$ collisions with respect to $\mathrm{p}+\mathrm{A}$ interactions has been suggested as a possible signature of the phase transition from hadronic matter to a QGP [5]. Many experiments at different accelerator facilities have comprehensively measured the yield of strange hadrons [6-13].

In this paper, we present the new experimental results on strangeness production, obtained from the fixed-target $\mathrm{Au}+\mathrm{Au}$ collisions at $\sqrt{\mathrm{s}_{\mathrm{NN}}}=4.5 \mathrm{GeV}$ and $\mathrm{Al}+\mathrm{Au} \sqrt{\mathrm{s}_{\mathrm{NN}}}=4.9 \mathrm{GeV}$, and compare with the previous results from different AGS experiments. In fixed-target collisions, the amount of energy deposited in the target nucleus can be determined. Another important issue in the field has been to quantify whether or not nucleus-nucleus collisions can be represented as a superposition of independent nucleon-nucleon collisions. In order to achieve this aim, a systematic measurement of Au- and Al-induced reactions on a Au target has been performed by the STAR experiment at RHIC. 
The comparison of the central to peripheral collisions for different target masses makes the space-time evolution of particle production and rescattering in hadronic matter more clear [14]. The measurements of strange hadron production in beam energy scan phase-I program (BES-I) has been reported in Refs. [15-19].

\section{Experimental data analysis}

Particles can be tracked and identified by the Time Projection Chamber (TPC) and Time-ofFlight (TOF) detectors at STAR. To demonstrate the capabilities of the STAR detector in fixedtarget mode, a fixed-target test run has been conducted for $\mathrm{Au}\left(\right.$ beam)+Au(target) collisions at $\sqrt{\mathrm{S}_{\mathrm{NN}}}$ $=4.5 \mathrm{GeV}$ and $\mathrm{Al}$ (beam) $+\mathrm{Au}$ (target) at $\sqrt{\mathrm{S}_{\mathrm{NN}}}=4.9 \mathrm{GeV}$. The target was placed at the edge of the TPC about $211 \mathrm{~cm}$ away from the interaction region to make use of the full tracking volume of the TPC. Approximately 1.3 million events for $\mathrm{Au}+\mathrm{Au}$ collisions at $\sqrt{\mathrm{s}_{\mathrm{NN}}}=4.5 \mathrm{GeV}$ and around 3.4 million events for $\mathrm{Al}+\mathrm{Au}$ collisions at $\sqrt{\mathrm{S}_{\mathrm{NN}}}=4.9 \mathrm{GeV}$ were collected with top $30 \%$ centrality triggers.

The signals and raw yields of the strange hadrons $\left(\mathrm{K}_{\mathrm{S}}^{0}\right.$ and $\left.\Lambda\right)$ are extracted by the invariant mass distribution reconstructed by their hadronic weak decay channels: $\mathrm{K}_{\mathrm{S}}^{0} \rightarrow \pi^{+} \pi^{-}$with branching ratio of $69.2 \%$ and $\Lambda \rightarrow p \pi^{-}$with branching ratio of $63.9 \%$. The decay daughters $\pi$ and $p$ are identified by the energy loss measured by the TPC of the STAR detector.

\section{Results and Discussions}

The data were divided into six rapidity bins of 0.25 unit width from $y=-1.75$ to $y=-0.25$. After applying the selection cuts and subtraction of rotational background, the invariant mass distribution for $\pi^{+} \pi^{-}$and $p \pi^{-}$shown in Figure 2 .
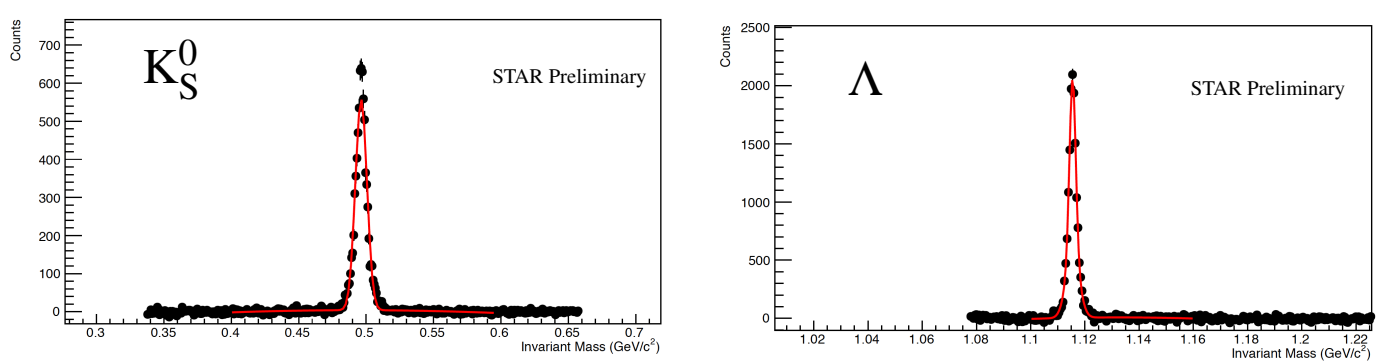

Figure 2: The $\pi^{+} \pi^{-}$(left) and $p \pi^{-}$(right) invariant mass distributions from the most central (0$5 \%) \mathrm{Al}+\mathrm{Au}$ collisions at $\sqrt{\mathrm{s}_{\mathrm{NN}}}=4.9 \mathrm{GeV}$

To determine the signal peak width as well as the shape of the remaining residual background, a superposition of the double-gaussian and polynomial functions was used. The raw spectra is then corrected for the reconstruction efficiency and geometrical acceptance, and we obtain the corrected $p_{\mathrm{T}}$ distribution for $\mathrm{K}_{\mathrm{S}}^{0}$ and $\Lambda$ in $\mathrm{Au}+\mathrm{Au}$ collisions at $\sqrt{\mathrm{s}_{\mathrm{NN}}}=4.5 \mathrm{GeV}$ and $\mathrm{Al}+\mathrm{Au}$ at $\sqrt{\mathrm{s}_{\mathrm{NN}}}=4.9$ $\mathrm{GeV}$. The efficiency corrected $p_{\mathrm{T}}$ spectra for $\mathrm{K}_{\mathrm{S}}^{0}$ and $\Lambda$ in different collision centrality class $0-5 \%$, $5-10 \%, 10-15 \%, 15-20 \%, 20-25 \%$ and $25-30 \%$ in Al+Au fixed-target collisions at $\sqrt{\mathrm{s}_{\mathrm{NN}}}=$ $4.9 \mathrm{GeV}$ are shown in Figure 3 and 4. 

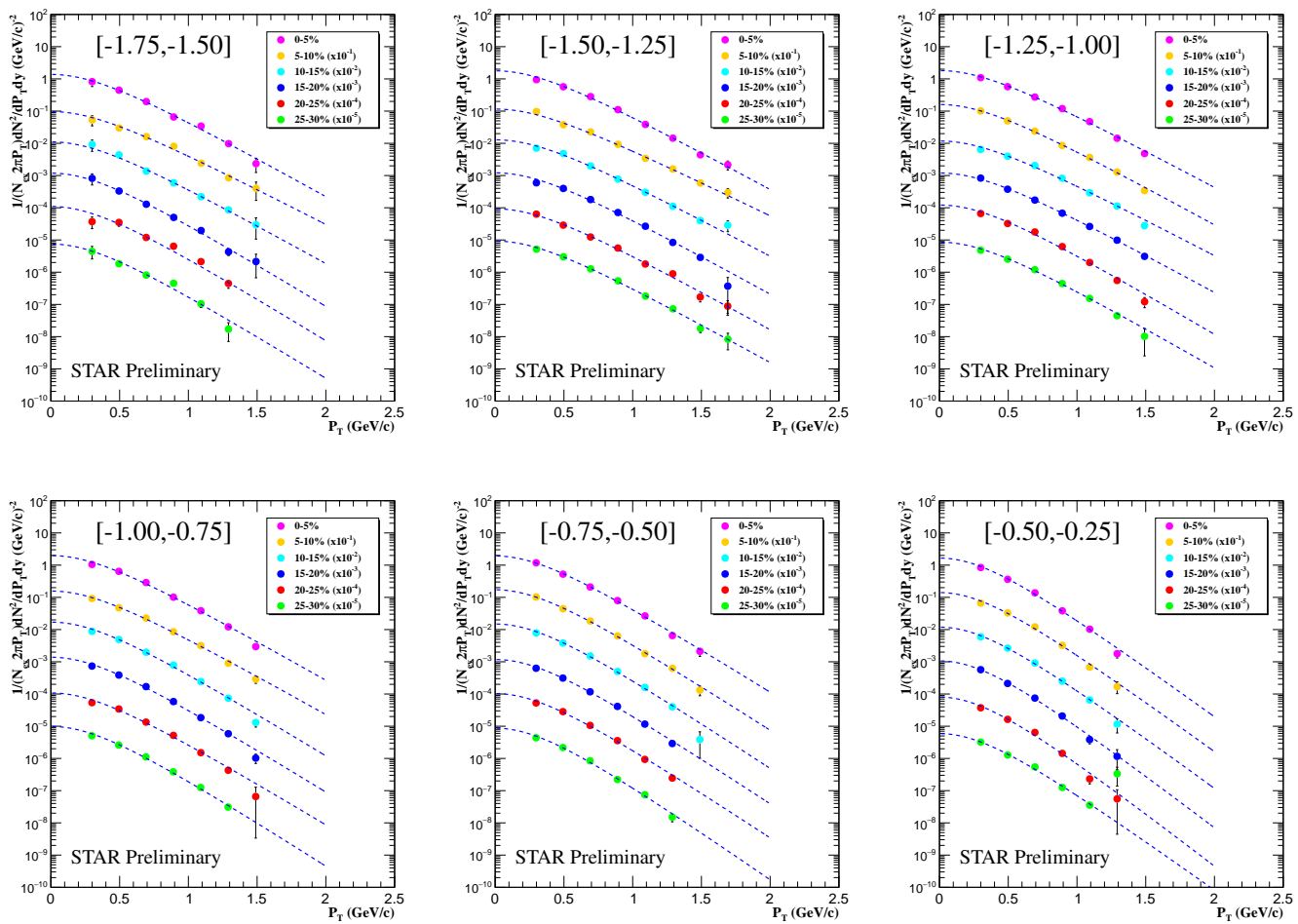

Figure 3: The $p_{\mathrm{T}}$ spectra of $\mathrm{K}_{\mathrm{S}}^{0}$ mesons for different rapidity ranges from STAR fixed-target $\mathrm{Al}+\mathrm{Au}$ collisions at $\sqrt{\mathrm{s}_{\mathrm{NN}}}=4.9 \mathrm{GeV}$. The data points are scaled by a factor of 10 for better visualization. Uncertainties are statistical only. The data are extrapolated to low and high $p_{\mathrm{T}}$ with the exponential fitting function.

The acceptance of the detector is limited at low $p_{\mathrm{T}}$ and is finite statistics at high $p_{\mathrm{T}}$, due to these limitations, spectra in these two regions were not measured, hence extrapolation to those regions is done with the fitting function in order to obtain the $p_{\mathrm{T}}$ integrated yield $(d N / d y)$. The range in $p_{\mathrm{T}}$ reflects the acceptance of the detector in different rapidity bins. The dashed line represents the best fits to the spectra. For $\mathrm{K}_{\mathrm{S}}^{0}$ the spectra are fitted and hence extrapolated with exponential function:

$$
\frac{d^{2} N}{2 \pi p_{T} d p_{T} d y} \propto e^{\frac{m_{T}}{T}}
$$

While $\Lambda$ spectra is fitted and extrapolated with the Boltzmann function:

$$
\frac{d^{2} N}{2 \pi p_{T} d p_{T} d y} \propto m_{T} e^{\frac{m_{T}}{T}}
$$

where $m_{\mathrm{T}}$ is transverse mass.

By integrating the $p_{\mathrm{T}}$ spectra, the integrated yield $(\mathrm{dN} / \mathrm{dy})$ is obtained in the measured $p_{\mathrm{T}}$ range and the extrapolated range with the fitting function at low and high $p_{\mathrm{T}}$.

Figure 5 (left) shows the STAR fixed-target $\mathrm{K}_{\mathrm{S}}^{0}$ rapidity density distribution (red) compared with the $K^{+}$and $K^{-}$from E917 experiment from AGS [20]. The data are reflected at midrapidity 

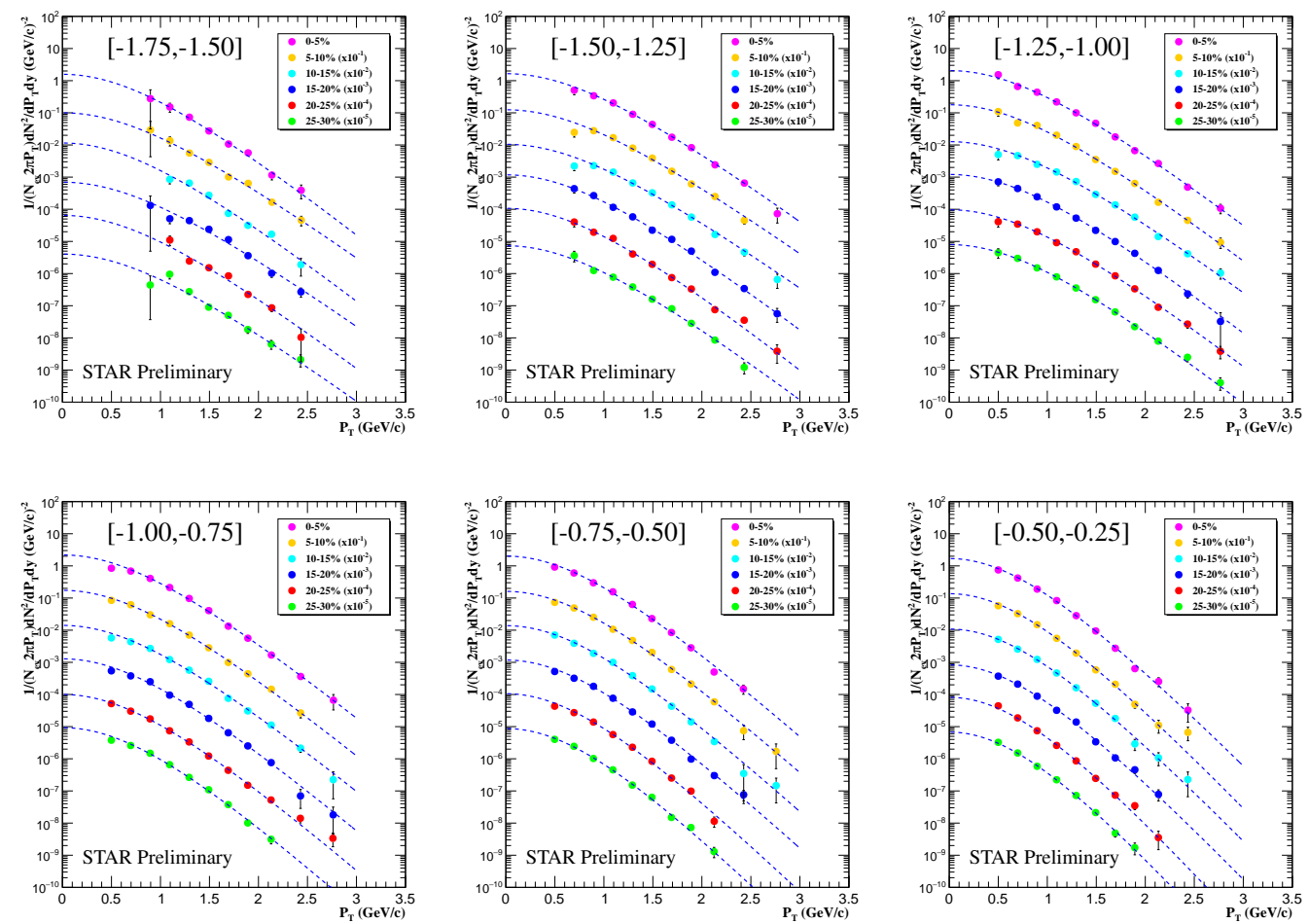

Figure 4: The $p_{\mathrm{T}}$ spectra of $\Lambda$ for different rapidity ranges from STAR fixed-target $\mathrm{Al}+\mathrm{Au}$ collisions at $\sqrt{\mathrm{S}_{\mathrm{NN}}}=4.9 \mathrm{GeV}$. The data points are scaled by a factor of 10 for better visualization. Uncertainties are statistical only. The data are extrapolated to low and high $p_{\mathrm{T}}$ with the Boltzman fitting function.

and plotted again as open symbols. The STAR $\mathrm{K}_{\mathrm{S}}^{0}$ yield agrees with average of E917 $\mathrm{K}^{+}$and $\mathrm{K}^{-}$ yield within uncertainties. Furthermore, the distributions do not appear to be Gaussian and there are even flatter distributions at lower energies. The flatness of the mid-rapidity points compared to the Gaussian distribution, makes it harder to extract the total yield, since there is a small variation within limited acceptance to constrain the shape. The reason why they might not be Gaussian is, at least in the cascade picture, $K^{+}$are predominately produced via associated production, i.e. $N N \rightarrow \Lambda K^{+} \mathrm{N}$, and so have residual correlations with the baryon number. In other pictures we can still imagine a residual correlations with baryon number, since the $K^{+}$has a light quark, not an antiquark in it, so will be correlated with baryon number. There is no reason that stopped baryons (or quarks) create a Gaussian distribution, and in fact the net protons are far from the Gaussian distribution depending on the energy. On the other hand negative kaons do not have any residual baryon number in them and they do appear to be narrower than the $K^{+}$, though again uncertainties make it hard to be definitive. $\mathrm{K}_{\mathrm{S}}^{0}$ on the other hand is the average of $K^{+}$and $K^{-}$[21]. Figure 5 (right) shows the STAR $\mathrm{K}_{\mathrm{S}}^{0}$ rapidity density comparison with the $K^{+}$and $K^{-}$from E802 experiment from AGS. Since $\mathrm{K}_{\mathrm{S}}^{0}$ is the average of $K^{+}$and $K^{-}$, so the $\mathrm{K}_{\mathrm{S}}^{0}$ from STAR lies between $K^{+}$and $K^{-}$from E802, shows consistency. The difference in center of mass energy between STAR and 

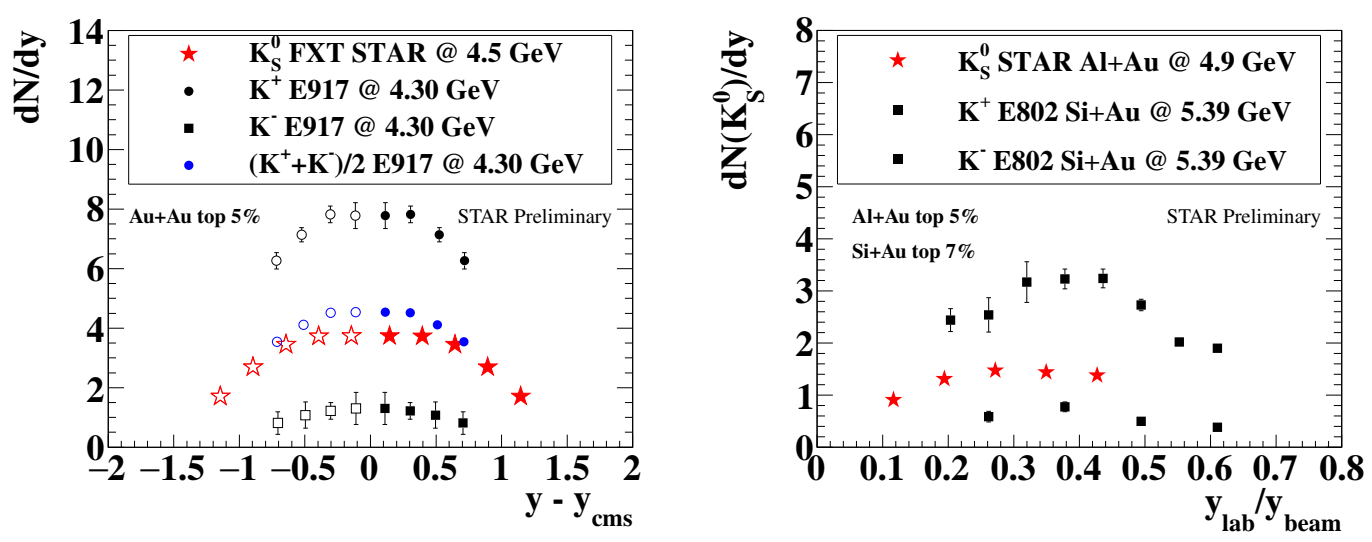

Figure 5: (Left) $\mathrm{K}_{\mathrm{S}}^{0}$ rapidity density (0-5\%) from the STAR fixed-target $\mathrm{Au}+\mathrm{Au}$ collisions $\sqrt{\mathrm{S}_{\mathrm{NN}}}$ $=4.5 \mathrm{GeV}$. STAR data are plotted as red stars. Open symbols are reflected around midrapidity. E917 points for $\mathrm{K}^{+}$and $\mathrm{K}^{-}$mesons are plotted for comparison in black. The blue points are the average of the $\mathrm{E} 917 \mathrm{~K}^{+}$and $\mathrm{K}^{-}$points. (Right) $\mathrm{K}_{\mathrm{S}}^{0}$ rapidity density $(0-5 \%)$ from the STAR fixedtarget $\mathrm{Al}+\mathrm{Au}$ collisions at $\sqrt{\mathrm{s}_{\mathrm{NN}}}=4.9 \mathrm{GeV}$ (red) plotted against the $K^{+}$and $K^{-}$(black) from E802. Uncertainties are statistical only.

E802, makes the $\mathrm{K}_{\mathrm{S}}^{0} d N / d y$ about $30 \%$ lower than the average of $K^{+}$and $K^{-}$. The center of mass energy for the STAR is $4.9 \mathrm{GeV}$ and for E802 experiment is $5.39 \mathrm{GeV}$. While figure 5 (left) is for the same particle species i.e. Au-induced reaction on Au target from the STAR and E917, while figure 5 (right) is for different particle species, Al-induced reaction on Au target from STAR and Si-induced reaction on Au target from E802 experiment. The $d N / d y$ distributions in the central $\mathrm{Au}+\mathrm{Au}$ collisions exhibits a "projectile-like" peak which is not seen in the extrapolation in $\mathrm{Al}+\mathrm{Au}$ collisions. Therefore, the peak position of the distribution for $K^{+}$and $K^{-}$and thus for $\mathrm{K}_{\mathrm{S}}^{0}$ shifts towards the lower rapidity for heavier projectile. An increased in $\mathrm{K}_{\mathrm{S}}^{0}$ yield is also observed from the light target to the heavier projectile.

Figure 6 shows the excitation function of the particle yield $(d N / d y)$ for $\mathrm{K}_{\mathrm{S}}^{0}$ and $\Lambda$ from the most central $(0-5 \%) \mathrm{Au}+\mathrm{Au}$ fixed-target collisions at $\sqrt{\mathrm{s}_{\mathrm{NN}}}=4.5 \mathrm{GeV}$ from STAR (red) scaled by the average number of participants, compared to the corresponding data from different AGS and CERN experiments. The data from STAR follows the similar trend established by AGS and CERN experiments.

\section{Summary}

We presented recent measurements of strange hadron $\left(\mathrm{K}_{\mathrm{S}}^{0}\right.$ and $\left.\Lambda\right)$ production from $\mathrm{Au}+\mathrm{Au}$ collisions at $\sqrt{\mathrm{s}_{\mathrm{NN}}}=4.5 \mathrm{GeV}$ and $\mathrm{Al}+\mathrm{Au}$ at $\sqrt{\mathrm{s}_{\mathrm{NN}}}=4.9 \mathrm{GeV}$ from the STAR Fixed-Target program. The $p_{\mathrm{T}}$ and $d N / d y$ spectra of the strange hadrons are in a good agreement with previously published results from the different AGS experiments. Strong target mass dependence is found for the multiplicity density in most central collisions. The shift in the peak position of the rapidity density distribution for $\mathrm{K}_{\mathrm{S}}^{0}$ towards the lower rapidity for heavier projectile is observed. The increase in $\mathrm{K}_{\mathrm{S}}^{0}$ yield is also observed from the light projectile to the heavier projectile. In general, there is a 


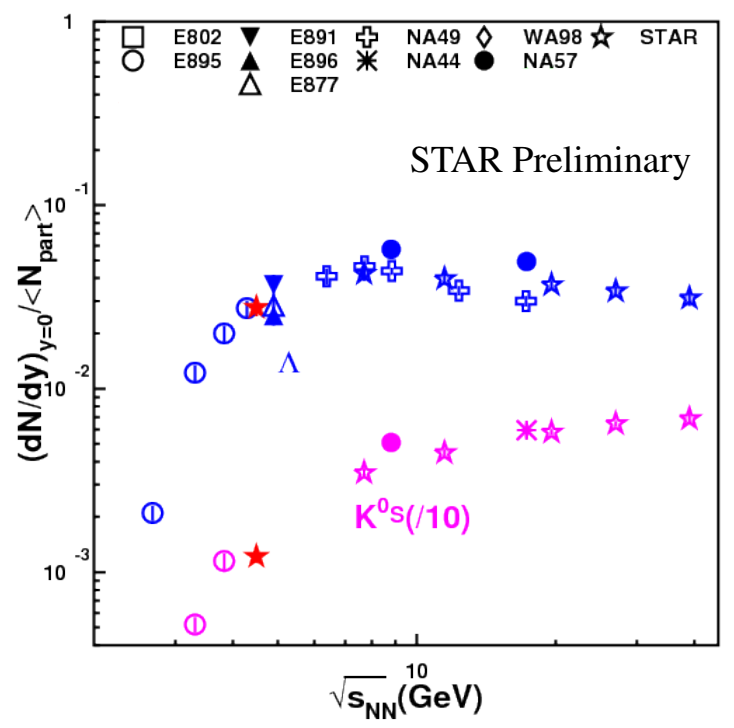

Figure 6: The excitation function of the integrated yield, dN/dy, at midrapidity, scaled by the average number of participants, for $\mathrm{K}_{\mathrm{S}}^{0}$ and $\Lambda$. The STAR fixed-target data, represented by red stars, are plotted alongside the data from several other experiments and are consistent with the trend. AGS and CERN experiments data is from [22-32].The $\mathrm{K}_{\mathrm{S}}^{0}$ points have been scaled by a factor of 10 to make the plot easier to read.

good agreement between the STAR data and the previously published data from different AGS and CERN experiments, which proves that STAR can be successfully operated in fixed-target mode.

\section{Acknowledgments}

Muhammad Usman Ashraf thanks the support by the National Natural Science Foundation of China (Grant No. 11622539, 11335005), the Major State Basic Research Development Program in China (No. 2014CB845400), and the Tsinghua University Initiative Scientific Research Program.

\section{References}

[1] E. Laermann et al. Nucl. Phys. A 610 (1996) 1c-12c

[2] J. Adams, et al. Nucl. Phys. A 757 (2005) 102-183.

[3] M. M. Aggarwal et al 20101007.2613 [nucl-ex]

[4] C. Alt et al. Phys. Rev. C 77 (2008) 024903.

[5] J. Rafelski and B. Muller 1982 Phys. Rev. Lett. 481066.

[6] P. Chung et al. Phys. Rev. Lett. 91 (2003) 202301.

[7] S. Albergo et al. Phys. Rev. Lett. 88 (2002) 062301.

[8] B.B. Back et al. Phys. Rev. Lett. 87 (2001) 242301.

[9] C. Alt et al. Phys. Rev. C 78 (2008) 034918. 
[10] F. Antinori et al. J. Phys. G 37 (2010) 045105.

[11] B.I. Abelev et al. Phys. Rev. C 77(2008), 044908.

[12] K. Adcox et al. Phys. Rev. Lett. 89 (2002) 092302.

[13] B. B. Abelev et al. Phys. Rev. Lett. 111 (2013) 222301.

[14] T. Abbott et al. Phys. Rev. C 50 (1994) 1024.

[15] F. Zhao (for the STAR Collaboration), AIP Conf. Proc. 1441 (2012) 797.

[16] X. Zhu (for the STAR Collaboration), Acta Phys. Pol. B Proc. Suppl. 5 (2012) 213.

[17] X. Zhu (for the STAR Collaboration), 1203.5182v1 [nucl-ex].

[18] X. Zhang (for the STAR Collaboration), Acta Phys. Pol. B Proc. Suppl. 5 (2012) 509.

[19] M. U. Ashraf (for the STAR Collaboration) J. Phys. Conf. Ser. 668 (2016) 012095.

[20] C. Ogilvie, Nucl. Phys. A 630 (1998) 571c.

[21] L. Ahle et al. Phys.Lett. B 490 (2000) 53-60.

[22] J. L. Klay et al. Phys. Rev. C 68 (2003) 054905.

[23] C. Pinkenburg (for the E895 collaboration) Nucl. Phys. A 698 (2002) 495c.

[24] L. Ahle et al. Nucl. Phys. A 610 (1996) 139c.

[25] J. Barrette et al. Phys. Rev. C 63 (2001) 014902.

[26] Ahmad. S. et al. Phys. Lett. B 382 (1996) 35.

[27] S. Albergo et al. Phys. Rev. Lett. 88 (2002) 062301.

[28] I. G. Bearden et al. Phys. Rev. C 66 (2002) 044907.

[29] M Gazdzicki (for the NA49 Collaboration) J. Phys. G 30 (2004) S701.

[30] T. Anticic et al. Phys. Rev. Lett. 93 (2004) 022302.

[31] F Antinori et al. J. Phys. G 32 (2006) 2065.

[32] M. M. Aggarwal et al. Phys. Rev. C 67 (2003) 014906. 